\title{
Freeze Drying Method with Gaseous Nitrogen for Biological Application of Helium Ion Microcopy
}

\section{Kunihiro Uryu ${ }^{1}$, Charles M. Rice ${ }^{2}$, Maria Teresa Catanese ${ }^{2,3}$, Gaetano Santulli ${ }^{4}$, Hana Totary- Jain $^{5}$, Chuong Huynh ${ }^{6}$, Bernhard Goetze ${ }^{6}$}

${ }^{1}$ Electron Microscopy Resource Center, ${ }^{2}$ Center for the Study of Hepatitis C, Laboratory of Virology and Infectious Disease, The Rockefeller University, New York, New York, USA; ${ }^{3}$ Department of Infectious Diseases, King's College London, London SE1 9RT, United Kingdom, ${ }^{4}$ Department of Physiology, Columbia University, New York, NY, 10032, ${ }^{5}$ Department of Molecular Pharmacology \& Physiology, University of South Florida, Morsani College of Medicine, Tampa, FL 33612, ${ }^{6}$ Carl Zeiss Microscopy, LLC., Ion Microscopy Innovation Center, MA 01960 USA

For examination on fine topographic features of biological samples, scanning electron microscopy (SEM) has been the major player. However, high resolution SEM imaging requires samples to be conductive and readily adaptable to a high vacuum environment. These require dehydration and conductive coating. Minute surface details of biological samples can be obscured by the metal coating. To deal with these challenges, the injecting variable nitrogen pressure, water vapor, environmental chamber were introduced. While these are useful, they compromise achievable resolution and signal to noise as a result of skirting beam. To take another approach Helium Ion Microscope (HIM) was introduced as a result of development of gas-field-ionization source, reduced aberrations and charge compensation electron flood gun. HIM can potentially be a super-resolution scanning microscope of detailed topographic architecture of biological organisms, because it demonstrated high resolution, high contrast, long focal depth, clean signal and no requirement of conductive coating. However, successful biological applications have been limited and it awaits further methodological supports ${ }^{1,2,3}$. Thus routine usage of HIM to examination of biological organizations awaits a sample preparation protocol for broad range of biological organisms.

We have made a series of attempts to come up with a reliable biological sample preparation method for HIM. Based on the understanding the nature of system and experiences from preliminary tests, we avoided metal, carbon or chemical coat/film that covers the surface, use of carbon sheet that is ready decomposed, and organic solvents to compromise lipid rich cell membrane. We aimed at 1) expose authentic cell surface, 2) avoid introduction of any contaminants as results of interaction with ion beam, and 3) maintain the best integrity of chemical and structure organization, especially cell membrane and associated structures. Based on these understanding, we came up with a three-step-procedure: 1) plunge freezing for instantaneous immobilization; 2) freeze drying in a temperature controlled $\left(<-100^{\circ} \mathrm{C}\right)$ chamber with a cold nitrogen gas (GN2) flow and desiccant; and 3) critical point drying (CPD) as a final dehydration step. Here we describe a new sample preparation procedure, coupled with a freeze dry method using gaseous nitrogen (FDGN2 method) resulting in unprecedented structural detail in HIM${ }^{4}$.

Now the present study presents the quality of HIM micrograms from wide range of biological organisms. FDGN2 method demonstrated significant capability to support HIM for its biological application. Using conventional methodology, various artifacts that were unnoticeable by regular SEMs turned out to be unavoidably visible by HIM. These include wash out cell membrane of cultured cell by EtOH (Figure a), thin film made of application of hexamethyldisilazane (HMDS) overlaying the cultured cell (Figure b), or decomposed carbon deposition over EM grid (Figure c). With the new FDGN2 method, highly preserved basic topographic architecture decorated with numerous villi $(\sim 70 \mathrm{~nm}$ 
in diameter) (Figure d,e) and fine meshwork with the identical size fibers (10-15 nm in diameter) appeared to the presentation of underneath structure (such as cytoskeleton) of cell lipid rich membrane (Figure e). Also clear visualization of the luminal surface of rat artery with red blood cells lying over endothelial cell (Figure f) and a red blood cell (Figure g). Biological samples with FDGN2 method made wide range of biological samples applicable to HIM and visualization of unprecedented structural features possible.
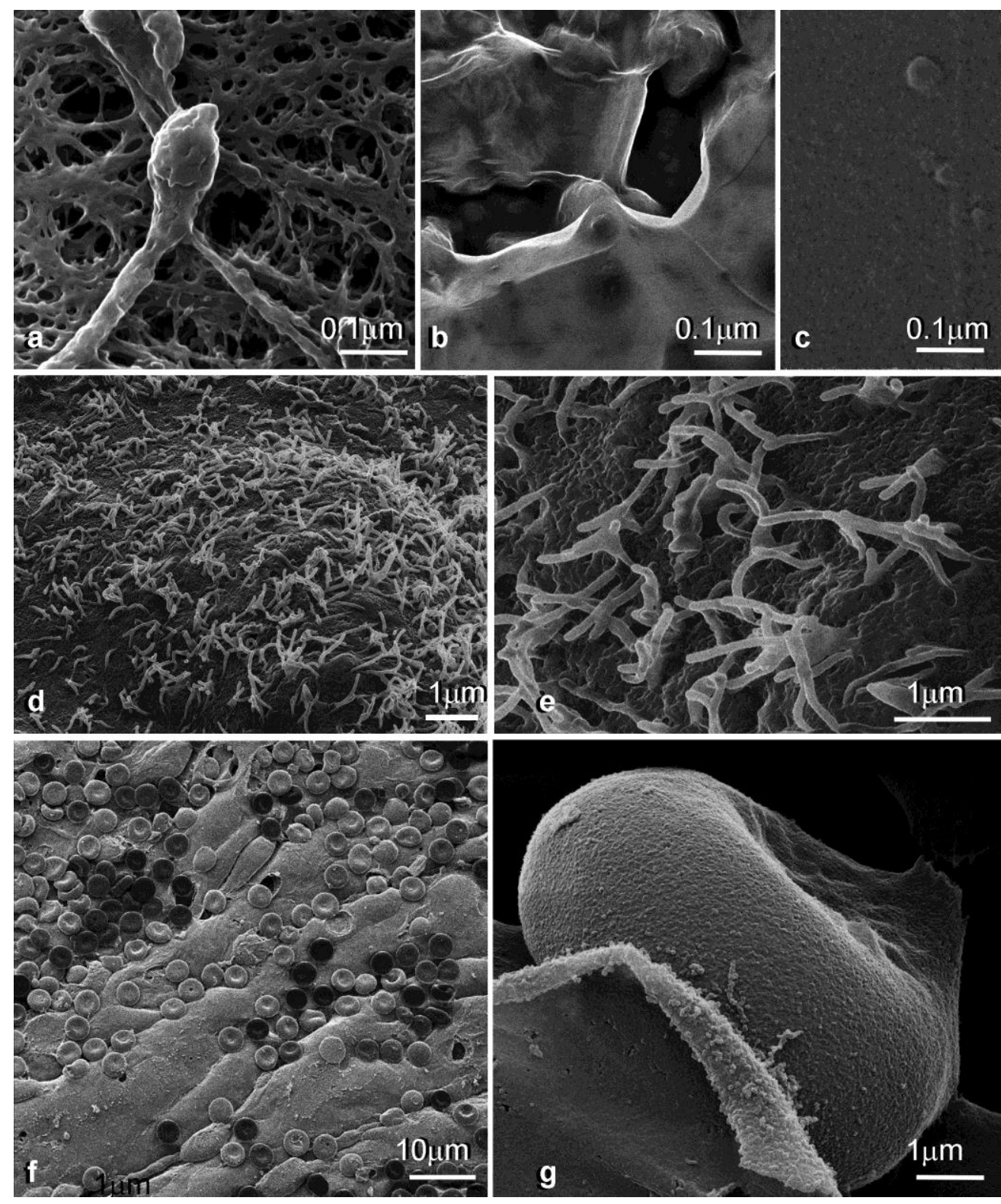

Reference: [1] Rice, W.L. et al. PloS one 8, e57051 (2013).

[2] Vanden Berg-Foels, W.S. Journal of microscopy 246, 168-176 (2012).

[3] Joens, M.S. et al. Sci. Rep. 3 (2013).

[4] Uryu et al., (2017) Microscopy and Microanalysis, 22, S3, P1142.
We offer this new methodology as an opportunity to surpass existing limitations in the study of biological structures, which in turn, will enhance our knowledge on the significance of biological organizations. The new knowledge could expand our vision and insight of complex bio-medical problems.

Figure: High power view of cultured cell membrane with ethanol dehydration and CPD (a), cultured cell membrane with ethanol dehydration and HMDS (b), carbon deposition on the surface of carbon coated EM grid (c), cultured cell with FDGN2 method (d), and its higher power (e). Luminal surface of rat artery with FDGN2 method (f), and a red blood cell (e).

Acknowledgements: The current study was supported by The Rockefeller University (K.U., C.R) 